\title{
3D Bipedal Walking with Knees and Feet: A Hybrid Geometric Approach
}

\author{
Ryan W. Sinnet and Aaron D. Ames
}

\begin{abstract}
Motivated by the goal of obtaining moreanthropomorphic walking in bipedal robots, this paper considers a hybrid model of a 3D hipped biped with feet and locking knees. The main observation of this paper is that functional Routhian Reduction can be used to extend twodimensional walking to three dimensions-even in the presence of periods of underactuation-by decoupling the sagittal and coronal dynamics of the 3D biped. Specifically, we assume the existence of a control law that yields stable walking for the 2D sagittal component of the 3D biped. The main result of the paper is that utilizing this controller together with "reduction control laws" yields walking in three dimensions. This result is supported through simulation.
\end{abstract}

\section{INTRODUCTION}

The consideration of a hipped biped with locking knees (as in [12]) and feet is an important step in understanding the intricacies of walking and, specifically, how to obtain moreanthropomorphic walking. Adding feet not only increases the postural stability of a biped, but also allows for a morenatural-looking and energy-efficient gait as shown in [6], [14], and [16]. The addition of feet, however, introduces new complications as an accurate model must take into account various transitions occurring throughout the biped's gait. Accordingly, we are confronted with a problem which can only be solved by modeling the biped as a complex hybrid system with multiple discrete domains (see Fig. 1).

The goal of this paper is to obtain walking for the 3D system by extending existing methods which give a stable gait for the 2D counterpart. Attaining a stable gait for a 2D model is difficult, and extending this to 3D only further complicates the problem. One must not only achieve a stable gait in the sagittal plane but also stabilize the walker in the coronal plane to prevent it from falling over-this has been done before for simpler biped models ([2], [4], [8], [17]). We consider a hipped biped with knees and feet with two degrees of rotational freedom at the ankles, i.e., the ankle can rotate in both the sagittal and coronal planes. Our idea is to effectively decouple the sagittal and coronal dynamicsas in [3] and [5] — such that we can stabilize the biped in the coronal plane while simultaneously applying an existing control law to the sagittal dynamics that yields $2 \mathrm{D}$ walking.

The main idea of this paper is to achieve this decoupling using reduction. We do this by exploiting the inherent symmetries present in the model (specifically in the coronal rotation angles) using geometric reduction. We consider a particular form of geometric reduction termed functional Routhian reduction developed in [1] and [2]. Like classical

R. W. Sinnet and A. D. Ames are with the Mechanical Engineering Department, Texas A\&M University, College Station, TX 77843 $\{$ rsinnet, aames $\}$ et amu. edu

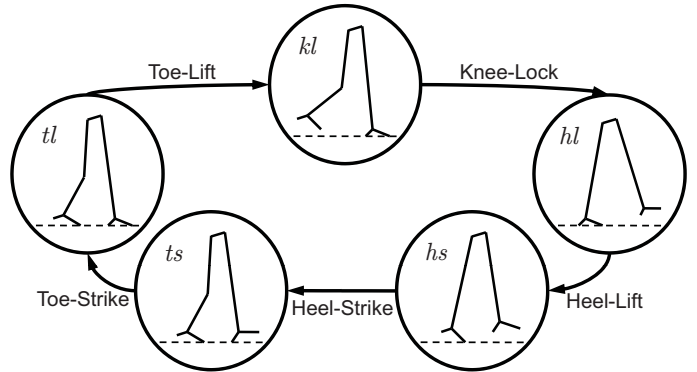

Fig. 1: Graph of system domains

reduction, we utilize the symmetries of "cyclic" variables, effectively removing these variables from the dynamics. The main difference between classical reduction and functional Routhian reduction is that we are able to set the conserved quantity to a function of the "cyclic" variables rather than a constant. This allows us to stabilize the walker while simultaneously decoupling the dynamics throughout the gait.

The main result of this paper is a control law which gives walking for the 3D model given a control law which gives stable walking for the reduced model (given in [14]). In order to use this 2D control law on our 3D model, we implement two additional control laws. The first of these control laws shapes the Lagrangian of the system to render it "almostcyclic". This allows us to perform functional Routhian reduction, which decouples the sagittal and coronal dynamics of the system and allows us to stabilize the walker in the coronal plane. However, reduction is only valid for certain initial conditions and so we implement an input/output linearization control law which stabilizes to the surface of conditions on which the reduction is valid. We verify through simulation that this control law results in walking.

\section{HYBRID SySTEMS AND CONSTRAINTS}

Throughout this paper, we use the notation and definitions given in full in [14]. A brief summary follows:

Hybrid systems are systems that display both continuous and discrete behavior and so bipedal walkers are naturally modeled by systems of this form: we consider a nonautonomous hybrid control system, which is a tuple $\mathscr{H} \mathscr{C}=$ $(\Gamma, D, U, G, R, F G)$. If there is no control, then we have a hybrid system $\mathscr{H}=(\Gamma, D, G, R, F)$ which is a hybrid control system with $U=\{0\}$.

The continuous component of the hybrid model will consist of dynamics dictated by Lagrangians. For a configuration space $Q \subseteq \mathbb{R}^{n}$, consider a Lagrangian given in coordinates by $L(q, \dot{q})=T(q, \dot{q})-V(q)$ with forcing term $\Upsilon$; the dynamics are obtained from the forced Euler-Lagrange equations:

$$
\mathcal{E L}_{q}(L)=\frac{d}{d t} \frac{\partial L}{\partial \dot{q}}-\frac{\partial L}{\partial q}=\Upsilon .
$$


Of special interest are Lagrangians of the form $L=$ $\frac{1}{2} \dot{q}^{T} M(q) \dot{q}-V(q)$ modeling mechanical systems, in which case the Euler-Lagrange equations become (cf. [11], §6.1.2):

$$
M(q) \ddot{q}+C(q, \dot{q}) \dot{q}+N(q)=\Upsilon
$$

which yields the control system:

$$
\begin{aligned}
f_{L}(q, \dot{q}) & =\left(\begin{array}{c}
\dot{q} \\
M^{-1}(q)(\Upsilon-C(q, \dot{q}) \dot{q}-N(q))
\end{array}\right) \\
g_{L}(q) & =\left(\begin{array}{c}
\mathbf{0}_{n \times k} \\
M^{-1}(q) B
\end{array}\right),
\end{aligned}
$$

where $\mathbf{0}_{n \times k}$ is a $n \times k$ matrix of zeros.

The discrete component of the hybrid system modeling a biped is obtained from various constraints: unilateral, holonomic and kinematic.

Simple discrete phenomena (such as knees locking and parts of the foot striking the ground) are modeled through unilateral constraints, written as tuples $\boldsymbol{h}=(Q, L, h)$; these yield corresponding domains and guards,

$$
\begin{aligned}
& D_{h}=\{(q, \dot{q}) \in T Q: h(q) \geq 0\}, \\
& G_{h}=\left\{(q, \dot{q}) \in T Q: h(q)=0 \text { and } d_{q} h(q) \dot{q}<0\right\},
\end{aligned}
$$

They also yield reset maps when viewed as simple kinematic constraints (to be defined shortly).

In some domains, we must impose a holonomic constraint, written as a tuple $\boldsymbol{\eta}=(Q, L, \eta)$ which yields a corresponding domain and guard,

$$
\begin{array}{r}
D_{\eta}=\left\{(q, \dot{q}, u) \in T Q \times \mathbb{R}^{k}: \lambda_{\eta}(q, \dot{q}, u) \geq 0\right\} \\
G_{\eta}=\left\{(q, \dot{q}, u) \in T Q \times \mathbb{R}^{k}: \lambda_{\eta}(q, \dot{q}, u)=0\right. \\
\text { and } \left.\dot{\lambda}_{\eta}(q, \dot{q}, u)<0\right\},
\end{array}
$$

and a reset map which is the identity map. These constraints also affect the dynamics: given a vector of Lagrange multipliers $\lambda_{\eta}: T Q \times \mathbb{R}^{k} \rightarrow \mathbb{R}^{c}$ with $k$ the number of inputs, one obtains the holonomically-constrained dynamics:

$M(q) \ddot{q}+C(q, \dot{q}) \dot{q}+N(q)+d_{q} \eta(q)^{T} \lambda_{\eta}(q, \dot{q}, u)=B u$,

which yields a control system as in (3) by solving for $\ddot{q}$.

In order to derive reset maps, we sometimes require additional constraints, kinematic constraints (containing the unilateral constraint, if present), which are written as tuples $\kappa=\left(Q_{s}, Q_{t}, M, \kappa, \iota, J\right)$ that give a reset map

$$
R\left(q_{s}, \dot{q}_{s}\right)=\left(\begin{array}{cc}
J & 0 \\
0 & J
\end{array}\right) \pi^{*}\left(\begin{array}{c}
\iota\left(q_{s}\right) \\
P\left(\iota^{*}\left(q_{s}, \dot{q}_{s}\right)\right)
\end{array}\right)
$$

with $P$ the rigid body plastic impact equations (cf. [14]).

\section{BIPEDAL MODEL}

In this section, the hybrid model of the biped that will be studied is introduced (note that due to space constraints, and the large complexity of the expressions, the specific expressions for the Lagrangians and constraint functions used to define this system are not included, but can be found at [18]). The coronal angles for all domains are shown in Fig. 2(f). We define the hybrid control system:

$$
\mathscr{H} \mathscr{C}_{3 \mathrm{D}}=(\Gamma, D, U, G, R, F G)
$$

with $\Gamma$ the oriented graph of the system (see Fig. 1):

$$
\begin{array}{r}
\Gamma=\left(\{k l, h l, h s, t s, t l\},\left\{e_{k l}=(k l, h l), e_{h l}=(h l, h s),\right.\right. \\
\left.\left.e_{h s}=(h s, t s), e_{t s}=(t s, t l), e_{t l}=(t l, k l)\right\}\right),
\end{array}
$$

The specific elements of (9) will now be given.

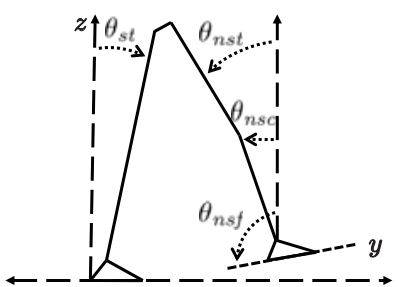

(a) Domain $1(\mathrm{kl})$

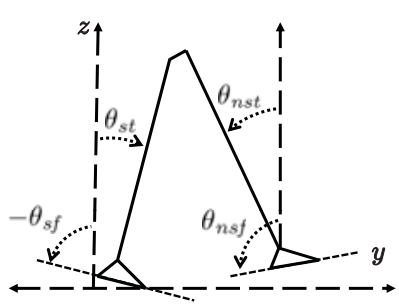

(c) Domain $3(h s)$

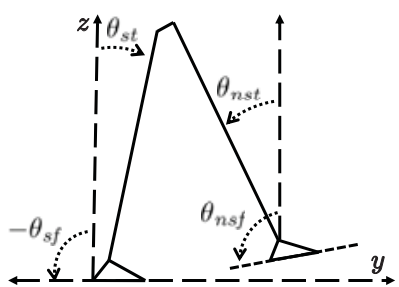

(b) Domain $2(h l)$

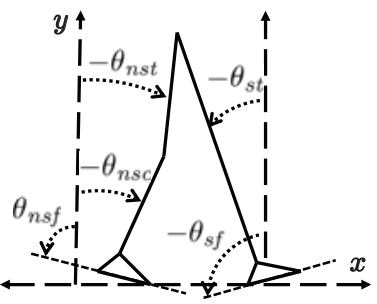

(d) Domain 4 (ts)

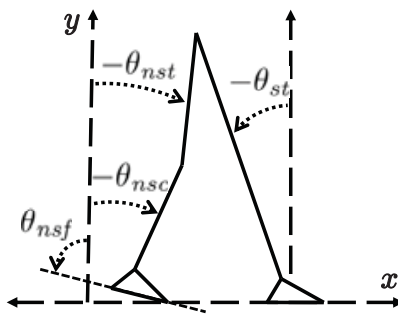

(e) Domain $5(t l)$

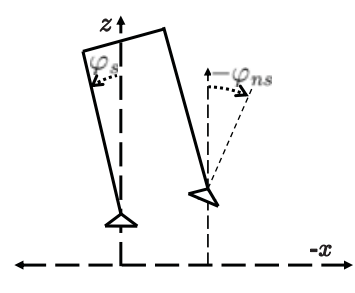

(f) Coronal angles
Fig. 2: Biped Configurations ( $\varphi$ angles not shown)

Domain $1(\mathrm{kl})$ : The stance foot is flat on the ground and the non-stance knee is unlocked. The configuration is shown in Fig. 2(a). The admissible control on this domain is $U_{k l}=\mathbb{R}^{6}$ and the guard is knee-lock, which occurs when the knee becomes straight and locks. We define the unilateral constraint $\boldsymbol{h}_{k l}=\left(Q_{k l}, L_{k l}, h_{k l}\right)$, where $Q_{k l}=$ $\mathbb{R}^{6}$ has coordinates $q_{k l}=\left(\theta_{s t}, \theta_{n s t}, \theta_{n s c}, \theta_{n s f}, \varphi_{s}, \varphi_{n s}\right)^{T}$, $L_{k l}\left(q_{k l}\right)=\frac{1}{2} \dot{q}_{k l}^{T} M_{k l}\left(q_{k l}\right) \dot{q}_{k l}-V_{k l}\left(q_{k l}\right)$ is the Lagrangian modeling the system, and $h_{k l}\left(q_{k l}\right)=\theta_{n s c}-\theta_{n s t}$ is the relative angle of the non-stance knee. Using $\boldsymbol{h}_{k l}$, the domain and guard are then given in (4).

We define the kinematic constraint $\boldsymbol{\kappa}_{k l}=$ $\left(Q_{k l}, Q_{h l}, M_{k l}, \kappa_{k l}, \iota_{k l}, J_{k l}\right)$, with $Q_{h l}$ introduced in the next domain, $\kappa_{k l}\left(q_{k l}\right)=h_{k l}\left(q_{k l}\right), \iota_{k l}: Q_{k l} \rightarrow Q_{k l} \cup J_{k l}^{-1}\left(Q_{h l}\right)$ the embedding given by $\left(\theta_{s t}, \theta_{n s t}, \theta_{n s c}, \theta_{n s f}, \varphi_{s}, \varphi_{n s}\right) \mapsto$ $\left(-\frac{\pi}{2}, \theta_{s t}, \theta_{n s t}, \theta_{n s c}, \theta_{n s f}, \varphi_{s}, \varphi_{n s}\right)$, and $J_{k l}=I$. The reset map is given by (8). The control system is as in (3).

Domain $2(h l)$ : Both knees are locked and the stance foot is flat on the ground. The configuration is shown in Fig. 2(b). The admissible control on this domain is $U_{h l}=\mathbb{R}^{5}$ and the guard is heel-lift. Therefore, we define the holonomic 
constraint $\boldsymbol{\eta}_{h l}=\left(Q_{h l}, L_{h l}, \eta_{h l}\right)$, where $Q_{h l}=\mathbb{R}^{6}$ has coordinates $q_{h l}=\left(\theta_{s f}, \theta_{s t}, \theta_{n s t}, \theta_{n s f}, \varphi_{s}, \varphi_{n s}\right)^{T}, L_{h l}\left(q_{h l}\right)=$ $\frac{1}{2} \dot{q}_{h l}^{T} M_{h l}\left(q_{h l}\right) \dot{q}_{h l}-V_{h l}\left(q_{h l}\right)$ is the Lagrangian modeling the system, and $\eta_{h l}: Q_{h l} \rightarrow \mathbb{R}$ is the height of the stance heel. Using $\boldsymbol{\eta}_{h l}$, the domain and guard are then given in (5).

The reset map is simply the identity map. Finally, the control system is given in (7) using the Lagrange multiplier $\lambda_{\eta_{h l}}\left(q_{h l}, \dot{q}_{h l}, u_{h l}\right)$ obtained from the holonomic constraint.

Domain $3(h s)$ : Both knees are locked and the system is rotating about the stance toe. The configuration is shown in Fig. 2(c). The admissible control on this domain is $U_{h s}=$ $\mathbb{R}^{5}$ and the guard is heel-strike. Therefore, we define the unilateral constraint $\boldsymbol{h}_{h s}=\left(Q_{h s}, L_{h s}, h_{h s}\right)$, where $Q_{h s}=$ $\mathbb{R}^{6}$ has coordinates $q_{h s}=\left(\theta_{s f}, \theta_{s t}, \theta_{n s t}, \theta_{n s f}, \varphi_{s}, \varphi_{n s}\right)^{T}$, $L_{h s}\left(q_{h s}\right)=\frac{1}{2} \dot{q}_{h s}^{T} M_{h s}\left(q_{h s}\right) \dot{q}_{h s}-V_{h s}\left(q_{h s}\right)$ is the Lagrangian modeling the system, and $h_{h s}\left(q_{h s}\right)$ is the height of the stance heel. Using $\boldsymbol{h}_{h s}$, the domain and guard are given in (4).

In order to define the reset map, we need the kinematic constraint, $\kappa_{h s}=\left(Q_{h s}, Q_{t s}, M_{t s}, \kappa_{h s}, \iota_{h s}, J_{h s}\right)$, with $Q_{t s}$ and $M_{t s}$ introduced in the next domain, $\kappa: Q_{h s} \rightarrow \mathbb{R}^{3}$ the non-stance heel position, and $\iota_{h s}: Q_{h s} \rightarrow Q_{h s} \cup J_{h s}^{-1}\left(Q_{t s}\right)$ the embedding given by $\left(\theta_{s f}, \theta_{s t}, \theta_{n s t}, \theta_{n s f}, \varphi_{s}, \varphi_{n s}\right) \mapsto$ $\left(\theta_{s f}, \theta_{s t}, \theta_{s t}, \theta_{n s t}, \theta_{n s f}, \varphi_{s}, \varphi_{n s}\right)$.

The reset map requires that the non-stance and stance legs be switched through a relabeling matrix $J_{h s}$ (consisting of 1 's and 0's) that flips the angles. The reset map is then given in (8). Finally, the control system is

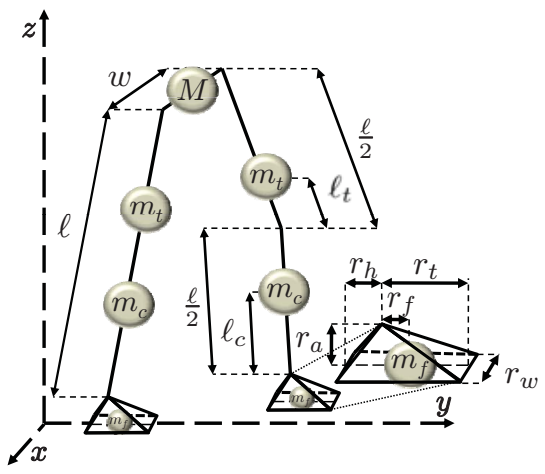

Fig. 3: Model configuration given in (3).

Domain 4 (ts): The non-stance knee is unlocked and the system is rotating about the stance heel. The non-stance toe is on the ground. The configuration is shown in Fig. 2(d). The admissible control on this domain is $U_{t s}=\mathbb{R}^{6}$ and the guard is toe-strike, which occurs when the stance toe rolls into the ground. Therefore, we define the unilateral constraint $\boldsymbol{h}_{t s}=\left(Q_{t s}, L_{t s}, h_{t s}\right)$, where $Q_{t s}=\mathbb{R}^{7}$ has coordinates $q_{t s}=\left(\theta_{s f}, \theta_{s t}, \theta_{n s t}, \theta_{n s c}, \theta_{n s f}, \varphi_{s}, \varphi_{n s}\right)^{T}$, $L_{t s}\left(q_{t s}\right)=\frac{1}{2} \dot{q}_{t s}^{T} M_{t s}\left(q_{t s}\right) \dot{q}_{t s}-V_{t s}\left(q_{t s}\right)$ is the Lagrangian modeling the system, and $h_{t s}\left(q_{t s}\right)$ is the height of the stance toe. Using $\boldsymbol{h}_{t s}$, the domain and guard are given in (4).

In order to define the reset map and control system, we will need to define the holonomic constraint, $\boldsymbol{\eta}_{t s}=$ $\left(Q_{t s}, L_{t s}, \eta_{t s}\right)$, with $\eta_{t s}: Q_{t s} \rightarrow \mathbb{R}^{3}$ the $x, y, z$ position of the non-stance toe which will be enforced by a vector of Lagrange multipliers, $\lambda_{\eta_{t s}}: T Q_{t s} \times U_{t s} \rightarrow \mathbb{R}^{3}$, of the form $\lambda_{\eta_{t s}}\left(q_{t s}, \dot{q}_{t s}, u_{t s}\right)$. Augmenting the dynamics in this way will prevent the non-stance toe from moving.

We define the kinematic constraint $\boldsymbol{\kappa}_{t s}=$
$\left(Q_{t s}, Q_{t l}, M_{t s}, \kappa_{t s}, \iota_{t s}, J_{t s}\right)$ with $Q_{t l}$ introduced in the next domain, $\kappa_{t s}: Q_{t s} \rightarrow \mathbb{R}^{4}$ given by $\kappa_{t s}\left(q_{t s}\right)=\left(h_{t s}, \eta_{t s}^{T}\right)^{T}$, and $\iota_{t s}$ the identity map. Note that including the holonomic constraints causes the non-stance toe to have zero velocity after impact. The reset map is given by (8) with $J_{t s}=I$. The control system is obtained from (7).

Domain $5(t l)$ : The stance foot is flat on the ground and the non-stance knee is unlocked. The non-stance toe is on the ground. The configuration is shown in Fig. 2(e). The admissible control on this domain is $U_{t l}=\mathbb{R}^{6}$ and the guard is toe-lift, which occurs when the non-stance toe lifts from the the ground. Therefore, we define the holonomic constraint $\boldsymbol{\eta}_{t l}=\left(Q_{t l}, L_{t l}, \eta_{t l}\right)$, where $Q_{t l}=\mathbb{R}^{6}$ has coordinates $q_{t l}=\left(\theta_{s t}, \theta_{n s t}, \theta_{n s c}, \theta_{n s f}, \varphi_{s}, \varphi_{n s}\right)^{T}, L_{t l}\left(q_{t l}\right)=$ $\frac{1}{2} \dot{q}_{t l}^{T} M_{t l}\left(q_{t l}\right) \dot{q}_{t l}-V_{t l}\left(q_{t l}\right)$ is the Lagrangian modeling the system, and $\eta_{t l}: Q_{t l} \rightarrow \mathbb{R}^{3}$ is a vector specifying the $x, y, z$ positions of the non-stance toe. From the holonomic constraints, we calculate a vector of Lagrange multipliers. We use $\left(\lambda_{\eta_{t l}}\right)_{3}$ (i.e., the constraining force in the $z$ direction) to specify the domain and guard as given in (5).

The reset map is simply the identity map. Finally, the control system is obtained from (7) using the Lagrange multiplier derived from $\eta_{t l}\left(q_{t l}\right)$.

\section{Functional Routhian Reduction}

In this section, we extend the functional Routhian Reduction of [2] to cover the case of $m$ cyclic variables with external forces. Specifically, we will consider the nonconservative forces $\Upsilon: T Q \rightarrow \mathbb{R}^{n}$ which do not depend on $\varphi, \dot{\varphi}$ and act only on the $\theta$ component of the corresponding Euler-Lagrange equations. Performing this reduction on the biped will allow us to decouple the sagittal and coronal dynamics and then achieve stable walking in three dimensions.

Almost-Cyclic Lagrangians. Consider a system with configuration space $Q=S \times \mathbb{R}^{m}$, where $S$ is called the shape space. Let the coordinates be represented by $q=\left(\theta^{T}, \varphi^{T}\right)^{T}$ with $\theta \in S$ and $\varphi \in \mathbb{R}^{m}$. A Lagrangian $L_{\lambda}: T S \times T \mathbb{R}^{m} \rightarrow \mathbb{R}$ is almost-cyclic if it takes the form

$$
\begin{aligned}
& L_{\lambda}(\theta, \varphi, \dot{\theta}, \dot{\varphi})= \\
& \frac{1}{2}\left(\begin{array}{cc}
\dot{\theta}^{T} & \dot{\varphi}^{T}
\end{array}\right) M_{\lambda}(\theta)\left(\begin{array}{c}
\dot{\theta} \\
\dot{\varphi}
\end{array}\right)-W_{\lambda}(\theta, \varphi, \dot{\theta})-V_{\lambda}(\theta, \varphi), \\
& M_{\lambda}(\theta)= \\
& \left(\begin{array}{cc}
M_{\theta}(\theta)+M_{\varphi, \theta}^{T}(\theta) M_{\varphi}^{-1}(\theta) M_{\varphi, \theta}(\theta) & M_{\varphi, \theta}^{T}(\theta) \\
M_{\varphi, \theta}(\theta) & M_{\varphi}(\theta)
\end{array}\right), \\
& W_{\lambda}(\theta, \varphi, \dot{\theta})=\lambda^{T}(\varphi) M_{\varphi}^{-1}(\theta) M_{\varphi, \theta}(\theta) \dot{\theta} \\
& V_{\lambda}(\theta, \varphi)=V_{\text {fct }}(\theta)-\frac{1}{2} \lambda^{T}(\theta) M_{\varphi}^{-1}(\theta) \lambda(\theta),
\end{aligned}
$$

for some $\lambda: \mathbb{R}^{m} \rightarrow \mathbb{R}^{m}$ with $M_{\theta}: S \rightarrow \mathbb{R}^{n-m \times n-m}$ and $M_{\varphi}: S \rightarrow \mathbb{R}^{m \times m}$ are positive definite symmetric.

Momentum maps. Reduction is based on the concept of conserved quantities specificed by a momentum map:

$$
J(\theta, \varphi, \dot{\theta}, \dot{\varphi})=\frac{\partial L_{\lambda}}{\partial \dot{\varphi}}(\theta, \varphi, \dot{\theta}, \dot{\varphi})=M_{\varphi, \theta}(\theta) \dot{\theta}+M_{\varphi}(\theta) \dot{\varphi}
$$


Unlike standard Routhian reduction, for functional Routhian reduction we set this map equal to a function $\lambda(\varphi)$.

Functional Routhians. For an almost-cyclic Lagrangian $L_{\lambda}$, define the functional Routhian $L_{\mathrm{fct}}: T S \rightarrow \mathbb{R}$ as

$$
L_{\mathrm{fct}}(\theta, \dot{\theta})=\left[L_{\lambda}(\theta, \varphi, \dot{\theta}, \dot{\varphi})-\lambda^{T}(\varphi) \dot{\varphi}\right]_{J(\theta, \varphi, \dot{\theta}, \dot{\varphi})=\lambda(\varphi)}
$$

Because $J(\theta, \varphi, \dot{\theta}, \dot{\varphi})=\lambda(\varphi)$ implies that

$$
\dot{\varphi}=M_{\varphi}^{-1}(\theta)\left(\lambda(\varphi)-M_{\varphi, \theta}(\theta) \dot{\theta}\right),
$$

by direct calculation the functional Routhian is given by:

$$
L_{\mathrm{fct}}(\theta, \dot{\theta})=\frac{1}{2} \dot{\theta}^{T} M_{\theta}(\theta) \dot{\theta}-V_{\mathrm{fct}}(\theta) .
$$

The Euler-Lagrange equations of $L_{\lambda}$ (in the presence of external forcing $\Upsilon(q, \dot{q}, t))$ yield the dynamical system

$$
\begin{aligned}
& f_{L_{\lambda}}(q, \dot{q}, t)= \\
& \quad\left(\begin{array}{c}
\dot{q} \\
-M_{\lambda}^{-1}(q)\left(\mathcal{E} \mathcal{L}_{q}\left(L_{\lambda}\right)-M_{\lambda}(q) \ddot{q}-\Upsilon(\theta, \dot{\theta}, t)\right)
\end{array}\right),
\end{aligned}
$$

with $\mathcal{E L}_{q}\left(L_{\lambda}\right)$ the Euler-Lagrange equations of $L_{\lambda}$; see (1). In addition, the vector field, $f_{L_{\mathrm{fct}}}$, corresponding to $L_{\mathrm{fct}}$ is given by (3). The solutions of these two systems are related in the following manner (in a way analogous to the classical Routhian reduction result, see [9], pp. 260).

Theorem 1: Let $L_{\lambda}$ be an almost-cyclic Lagrangian with $m$ almost-cyclic variables and $L_{\mathrm{fct}}$ the corresponding functional Routhian with shape space $S=\mathbb{R}^{n-m}$. Additionally, let $\Upsilon: T S \times \mathbb{R} \rightarrow \mathbb{R}^{n}$ represent external forces applied to the system satisfying:

(i) $\Upsilon(\theta, \dot{\theta}, t)$ does not depend on $\varphi, \dot{\varphi}$,

(ii) $\Upsilon_{i}(\theta, \dot{\theta}, t)=0$ for $i=n-m+1, \ldots, n$.

(i.e., no external forces act on the angles of the almost-cyclic variables.)

Then, $(\theta(t), \varphi(t), \dot{\theta}(t), \dot{\varphi}(t))$ is a solution to the forced vector field $f_{L_{\lambda}}$ given by (14) on $\left[t_{0}, t_{F}\right]$ with

$$
\dot{\varphi}\left(t_{0}\right)=M_{\varphi}^{-1}\left(\theta\left(t_{0}\right)\right)\left(\lambda\left(\varphi\left(t_{0}\right)\right)-M_{\varphi, \theta}\left(\theta\left(t_{0}\right)\right) \dot{\theta}\left(t_{0}\right)\right),
$$

if and only if $(\theta(t), \dot{\theta}(t))$ is a solution to the forced vector field $f_{L_{\mathrm{fct}}}$ given by (3) and $(\varphi(t), \dot{\varphi}(t))$ satisfies:

$$
\dot{\varphi}(t)=M_{\varphi}^{-1}(\theta(t))\left(\lambda(\varphi(t))-M_{\varphi, \theta}(\theta(t)) \dot{\theta}(t)\right) .
$$

This theorem is the main theoretical result of this paper. The proof can be found in [15].

\section{Reduction CONTROL Laws}

In this section, we introduce the control laws that will be applied to the model considered in this paper. We assume the presence of a control law, $K_{2 \mathrm{D}}^{\delta}(\theta, \dot{\theta}, t)$, (with $\delta$ a set of control gains) that gives stable walking on the sagitallyrestricted model (the control law used is described in [14]).

The Lagrangian of the 3D biped considered in this paper has the general form (on each domain):

$$
\begin{aligned}
& L_{3 \mathrm{D}}(q, \dot{q})=-V_{3 \mathrm{D}}(q)+ \\
& \quad \frac{1}{2}\left(\begin{array}{ll}
\dot{\theta}^{T} & \dot{\varphi}^{T}
\end{array}\right) \underbrace{\left(\begin{array}{cc}
M_{\theta}(\theta) & M_{\varphi, \theta}^{T}(\theta, \varphi) \\
M_{\varphi, \theta}(\theta, \varphi) & M_{\varphi}(\theta, \varphi)
\end{array}\right)}_{M_{3 \mathrm{D}}(q)}\left(\begin{array}{c}
\dot{\theta} \\
\dot{\varphi}
\end{array}\right),
\end{aligned}
$$

where the inertia matrix $M_{3 \mathrm{D}}(q)$ has been written in block form and $V_{3 \mathrm{D}}(q)$ is the potential energy. The control system associated to this Lagrangian, $\left(f_{3 \mathrm{D}}, g_{3 \mathrm{D}}\right)$, is given in (3).

Reduced dynamics. Consider the sagittal dynamics of the $3 \mathrm{D}$ biped. These dynamics have configuration space $Q_{2 \mathrm{D}}=$ $\mathbb{R}^{n-m}$ (where $m$ is the number of cyclic variables in the 3D system) with coordinates $\theta$. The Lagrangian is given by

$$
L_{2 \mathrm{D}}(\theta, \dot{\theta})=\frac{1}{2} \dot{\theta}^{T} M_{\theta}(\theta) \dot{\theta}-V_{2 \mathrm{D}}(\theta),
$$

with $M_{\theta}(\theta)$ a submatrix as defined in (17) and $V_{2 \mathrm{D}}(\theta)=$ $\left.V_{3 \mathrm{D}}(q)\right|_{\varphi=0}$. These equations yield the control system $\left(f_{2 \mathrm{D}}, g_{2 \mathrm{D}}\right)$ as in (3). Applying the existing control law, $K_{2 \mathrm{D}}^{\delta}(\theta, \dot{\theta}, t)$, yields the dynamical system

$$
f_{2 \mathrm{D}}^{\delta}(\theta, \dot{\theta}, t)=f_{2 \mathrm{D}}(\theta, \dot{\theta})+g_{2 \mathrm{D}}(\theta) K_{2 \mathrm{D}}^{\delta}(\theta, \dot{\theta}, t) .
$$

This will later be specialized to each domain.

Having defined the 3D system and its reduced 2D counterpart, we turn our focus to shaping the 3D system in such a way that we can apply Theorem 1, making it equivalent to the $2 \mathrm{D}$ system. In an attempt to satisfy the conditions of Theorem 1, we implement two control laws. The first control law transforms the 3D Lagrangian, $L_{3 \mathrm{D}}(q, \dot{q})$, given by (10) into an almost-cyclic Lagrangian as in the statement of Theorem 1. The second control law uses input/output linearization to stabilize to the surface of initial conditions for which the reduction is valid. Combining these control laws with the assumed 2D control law, $K_{2 \mathrm{D}}^{\delta}(\theta, \dot{\theta}, t)$, allows us to achieve stable walking for the 3D biped.

Lagrangian shaping controller. Having an almost-cyclic Lagrangian enables us to perform reduction. This controller, therefore, shapes the Lagrangian of the system into an almost-cyclic Lagrangian. Consider the almost-cyclic Lagrangian $L_{\alpha}$ given in (10), where we choose the function $\lambda(\varphi)=-\alpha \varphi$, with $\alpha \in \mathbb{R}$ a positive constant specifying the rate of convergence, and switch the subscripts accordingly to represent the new control gain, $\alpha$.

Let $K_{3 \mathrm{D}}^{\delta}(\theta, \dot{\theta}, t)=\left(\left(K_{2 \mathrm{D}}^{\delta}(\theta, \dot{\theta}, t)\right)^{T}, \mathbf{0}_{m}\right)^{T}$ represent the 2D walking controller in a form which can be applied to the 3D system by padding the control vector with $m$ zeros representing the control inputs on the $m$ cyclic variables. Then define the feedback control law:

$$
\begin{aligned}
& K_{3 \mathrm{D}}^{\alpha, \delta}(q, \dot{q}, t)=B^{-1}\left(C_{3 \mathrm{D}}(q, \dot{q}) \dot{q}+N_{3 \mathrm{D}}(q)\right. \\
& \quad+M_{3 \mathrm{D}}(q) M_{\alpha}^{-1}(q)\left(K_{3 \mathrm{D}}^{\delta}(\theta, \dot{\theta}, t)-\mathcal{E L}_{q}\left(L_{\alpha}\right)+M_{\alpha}(q) \ddot{q}\right) .
\end{aligned}
$$

with $M_{\alpha}(q)$ the shaped inertia matrix as given in (11), $C_{\alpha}(q, \dot{q})$ the shaped Coriolis matrix, and $N_{\alpha}(q)=\frac{\partial V_{\alpha}(q)}{\partial q}$ with $V_{\alpha}(q)$ from (11) having the potential energy of the 2D system $V_{\text {fct }}(q)=V_{2 \mathrm{D}}(\theta)$. This yields the dynamical system

$$
f_{3 \mathrm{D}}^{\alpha, \delta}(q, \dot{q}, t)=f_{3 \mathrm{D}}(q, \dot{q})+g_{3 \mathrm{D}}(q) K_{3 \mathrm{D}}^{\alpha, \delta}(q, \dot{q}, t) .
$$

By Theorem 1, we have:

Proposition 1: $(\theta(t), \varphi(t), \dot{\theta}(t), \dot{\varphi}(t))$ is a solution to the vector field $f_{3 \mathrm{D}}^{\alpha, \delta}(q, \dot{q})$ on $\left[t_{0}, t_{F}\right]$ with

$$
\dot{\varphi}\left(t_{0}\right)=-M_{\varphi}^{-1}\left(\theta\left(t_{0}\right)\right)\left(\alpha \varphi\left(t_{0}\right)+M_{\varphi, \theta}\left(\theta\left(t_{0}\right)\right) \dot{\theta}\left(t_{0}\right),\right.
$$


if and only if $(\theta(t), \dot{\theta}(t))$ is a solution to the vector field $f_{2 \mathrm{D}}^{\delta}(\theta, \dot{\theta})$ and $(\varphi(t), \dot{\varphi}(t))$ satisfies:

$$
\dot{\varphi}(t)=-M_{\varphi}^{-1}(\theta(t))\left(\alpha \varphi(t)+M_{\varphi, \theta}(\theta(t)) \dot{\theta}(t)\right) .
$$

Thus for initial conditions satisfying (21), the dynamics of $f_{3 \mathrm{D}}^{\alpha, \delta}(q, \dot{q})$ can be effectively decoupled into the sagittal and coronal dynamics with the control law $K_{3 \mathrm{D}}^{\alpha, \delta}(q, \dot{q}, t)$. Furthermore, the coronal dynamics evolve according to (22).

Input-output linearization controller. Reduction allows us to effectively decouple the sagittal and coronal dynamics but only when the conditions (21) of Proposition 1 are satisfied. Since these conditions are not always satisfied we introduce a second control law to drive the system to the surface where these conditions are satisfied. We will use the standard method of input/output linearization (See [13] for the continuous case and [7], [10] for the hybrid analogue).

We begin by defining a new control system, $\left(f_{3 \mathrm{D}}^{\alpha, \delta}, g_{3 \mathrm{D}}^{\alpha, \delta}\right)$, with $f_{3 \mathrm{D}}^{\alpha, \delta}$ given in (20) and $g_{3 \mathrm{D}}^{\alpha, \delta}$ given by

$$
g_{3 \mathrm{D}}^{\alpha, \delta}(q)=g_{3 \mathrm{D}}(q)\left(\begin{array}{cc}
\mathbf{0}_{n-m \times n-m} & \mathbf{0}_{n-m \times m} \\
\mathbf{0}_{m \times n-m} & I_{m}
\end{array}\right) .
$$

Define the output

$$
y(q, \dot{q})=\dot{\varphi}+M_{\varphi}^{-1}(\theta)\left(\alpha \varphi+M_{\varphi, \theta}(\theta) \dot{\theta}\right) .
$$

If $y(q, \dot{q})=0$ then (21) is satisfied. Thus, we would like to drive the system to the surface

$$
\mathcal{Z}=\left\{\left(\begin{array}{c}
q \\
\dot{q}
\end{array}\right) \in T Q: y(q, \dot{q})=0\right\} .
$$

With this in mind and motivated by the standard method of input/output linearization, define the feedback control law:

$$
\begin{aligned}
& K_{3 \mathrm{D}}^{\epsilon, \alpha, \delta}(q, \dot{q}, t)= \\
& \quad-\left(\mathcal{L}_{g_{3 \mathrm{D}}^{\alpha, \delta}} y(q, \dot{q})\right)^{-1}\left(\mathcal{L}_{f_{3 \mathrm{D}}^{\alpha, \delta}} y(q, \dot{q})+\frac{1}{\epsilon} y(q, \dot{q})\right),
\end{aligned}
$$

where $\mathcal{L}$ is the Lie derivative. Note that it can be verified numerically that $\mathcal{L}_{g_{3 \mathrm{D}}^{\alpha, \delta}} y(q, \dot{q}) \neq 0$. Applying this control law gives us the dynamical system:

$$
\begin{gathered}
f_{3 \mathrm{D}}^{\epsilon, \alpha, \delta}(q, \dot{q}, t)=f_{3 \mathrm{D}}^{\alpha, \delta}(q, \dot{q}, t)+g_{3 \mathrm{D}}(q) K_{3 \mathrm{D}}^{\epsilon, \alpha, \delta}(q, \dot{q}, t) \\
\text { VI. Control LAW CONSTRUCTION }
\end{gathered}
$$

We now describe how the control laws of the previous section are applied to the our model, (9). We again assume the presence of a control law, $K_{2 \mathrm{D}, i}^{\delta}(\theta, \dot{\theta}, t)$ with corresponding $K_{3 \mathrm{D}, i}^{\delta}(q, \dot{q}, t), i \in V$, which causes stable walking in the reduced model. Our goal is to apply this control law in addition to applying reduction, whenever possible.

Note we only have full actuation of the $\varphi$ coordinates in domains $k l$ and $h l$ and thus can only apply reduction in these domains. Therefore, for $i \in\{h s, t s, t l\}$,

$$
f_{3 \mathrm{D}, i}^{\delta}(q, \dot{q}, t)=f_{3 \mathrm{D}, i}(q, \dot{q})+g_{3 \mathrm{D}, i}(q) K_{3 \mathrm{D}, i}^{\delta}(\theta, \dot{\theta}, t) .
$$

Note that sagittal control law implementation is not covered in this paper but is exactly analogous to [14].

Domain $1(\mathrm{kl})$ and Domain $2(h l)$ : We implement both $K_{3 \mathrm{D}, k l}^{\delta}(\theta, \dot{\theta}, t)$ and the reduction control laws for Domains
1 and 2; the implementations are the same so we cover both domains simultaneously. Let $i \in\{k l, h l\}$. We start with the control law $K_{3 \mathrm{D}, i}^{\alpha, \delta}(q, \dot{q}, t)$ given in (19) which gives the vector field $f_{3 \mathrm{D}, i}^{\alpha, \delta}(q, \dot{q}, t)$ (as in (20)). Using (23), we get the control system $\left(f_{3 \mathrm{D}, i}^{\alpha, \delta}, g_{3 \mathrm{D}, i}^{\alpha, \delta}\right)$. Finally, we implement $K_{3 \mathrm{D}, i}^{\epsilon, \alpha, \delta}(q, \dot{q}, t)$ as in (25) which gives the dynamical system:

$$
f_{3 \mathrm{D}, i}^{\epsilon, \alpha, \delta}(q, \dot{q}, t)=f_{3 \mathrm{D}, i}^{\alpha, \delta}(q, \dot{q}, t)+g_{3 \mathrm{D}, \mathrm{i}}^{\alpha, \delta}(q) K_{3 \mathrm{D}, i}^{\epsilon, \alpha, \delta}(q, \dot{q}) .
$$

\section{Simulation Results}

In this section, we present the simulation results for the bipedal model and control laws considered in this paper. We describe the setup and the resulting stable gait. The physical parameters can be found at [18]. We start in domain $k l$ on the guard $G_{k l}$ with our initial condition as the fixed point:

$$
\begin{aligned}
& \left(q_{k l}\right)_{0}=\left(\begin{array}{llll}
0.000874, & 0.000312, & 0.0341,
\end{array}\right. \\
& -0.278, \quad-0.278, \quad-1.847 \quad)^{T}, \\
& \left(\dot{q}_{k l}\right)_{0}=\left(\begin{array}{rrr}
-0.00641, & -0.00205, & 0.601, \\
0.293, & -7.284, & -7.181
\end{array}\right)^{T} .
\end{aligned}
$$

A sample gait is shown in Fig. 4 and a video can be found at [18]. First, note that the majority of the gait is spent in domains $k l$ and $h l$. In these domains, we perform reduction and thus we are able to implement a sagittal control law using full actuation. Note further that the biped does not fall over sideways - a result of the application of reduction-even though coronal stabilization is not present throughout the entire gait. Thus, we are able to achieve decoupled dynamics even with periods of underactuation.

Because the foot rotation in the coronal plane is small (see Fig. 5(c)), we make the simplifying assumption that the foot has no coronal rotation when it strikes the ground. This greatly reduces the complexity of the model without significantly affecting its validity. An additional consequence of ignoring the foot rotation is that the width of the foot is irrelevant as long as it is not so thin that coronal actuation of the ankle causes the foot to roll on its side. In our model, we use feet wide enough to negate this concern.

The phase portraits of the various angles are shown in Fig. 5. It is (numerically) clear that the control laws introduced in this paper resulted in a limit cycle, i.e., a walking gait for the biped. Note that the limit cycle of the system must go through 10 domains in order to complete one cycle (as

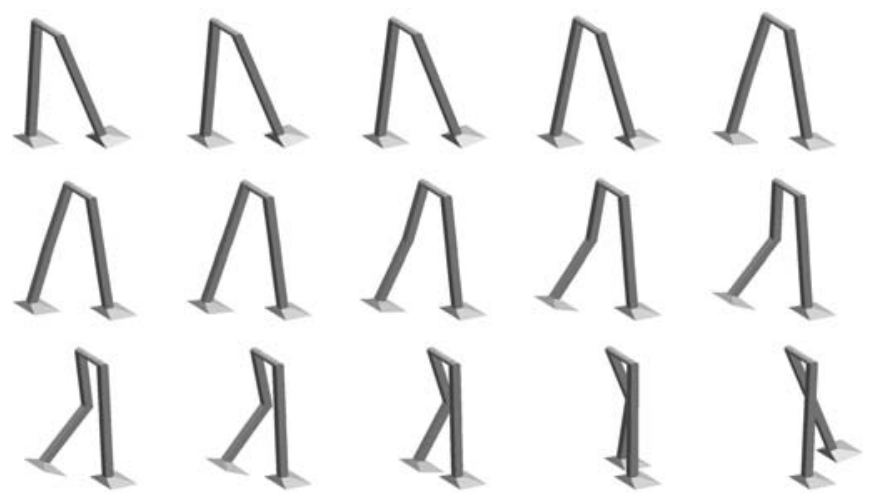

Fig. 4: Example of 3D gait 


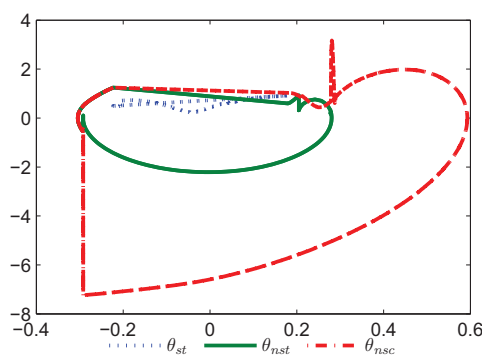

(a) Phase portrait of legs

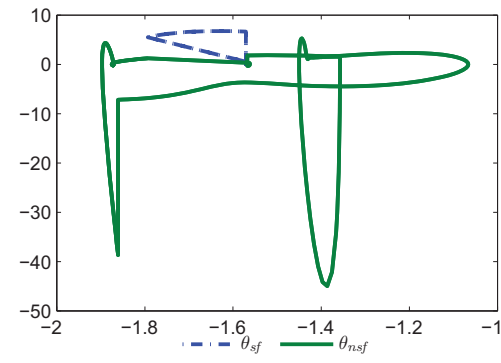

(b) Phase portrait of feet (sagittal plane)

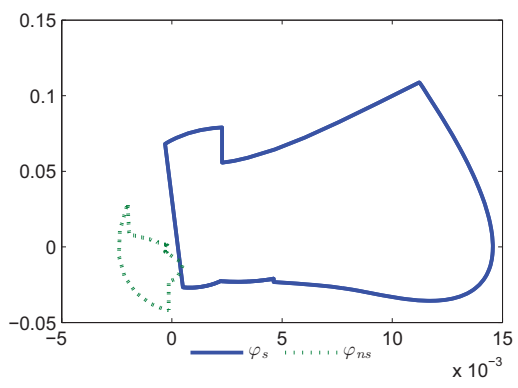

(c) Phase portrait of feet (coronal plane)

Fig. 5: Phase portraits

opposed to five domains in [14]); in other words, the system is bi-periodic. This is caused by the coronal swaying of the system, which occurs as a result of the back toe kicking off and to a lesser extent, the impact of the non-stance foot and the reduction controller. This swaying motion is beneficial in that it allows for extra clearance of the non-stance foot.

Note the
similarity
between Figs.
$5(\mathrm{a})$ and
$5(\mathrm{~b})$ and the
corresponding
figures in
[14]. The
phase portraits
are virtually
identical as we

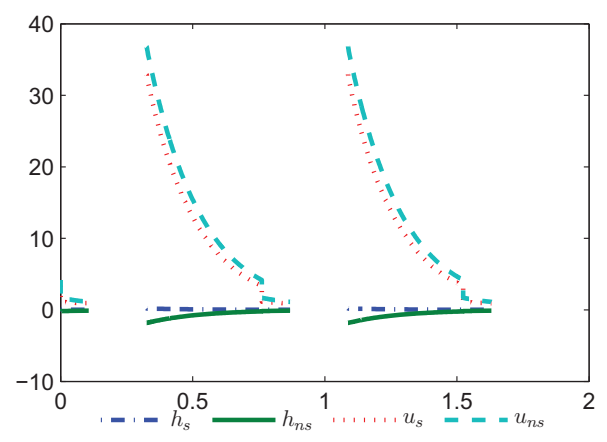

Fould expect 6: Virtual outputs and control
and hope. Thus reduction is effective at decoupling the sagittal and coronal dynamics of the system with the end result that it is possible to construct a hipped biped and utilize the much simpler 2D control laws of the reduced counterpart to effect stable walking.

In order for reduction to be valid, we needed to satisfy the conditions given in (21), i.e., stabilize to the surface (24) which was achieved through input/output linearization. This controller drives the system to this surface at an exponential rate as seen in Fig. 6. Additionally, the required amount of control decreases as the system gets closer to the surface. The gaps in the figure represent the domains in which we do not apply reduction. Also note that the zero dynamics are not invariant as evidenced by the jumps resulting from impacts.

The model considered is very complex. As a result, we were unable to calculate accurate eigenvalues of a linearized Poincaré map. Performing this calculation requires a high degree of numerical precision and as such, the calculation would require significant computing power. Thus, we can only state the gait appears to be stable as it does not diverge even after hundreds of steps and initial conditions near the limit cycle converge to the limit cycle. Future research should therefore include computing accurate eigenvalues to examine the stability of the system. Additionally, we would like to optimize the control gains used for reduction.

\section{REFERENCES}

[1] A. D. Ames and S. Sastry, "Hybrid Routhian reduction of hybrid Lagrangians and Lagrangian hybrid systems," in 25th American Control Conference, Minneapolis, MN, 2006.

[2] A. D. Ames, R. W. Sinnet, and E. D. B. Wendel, "Three-dimensional kneed bipedal walking: A hybrid geometric approach," in 12th International Conference on Hybrid Systems: Computation and Control, San Francisco, CA, 2009.

[3] C. Chevallereau, J. W. Grizzle, and C. Shih, "Asymptotically stable walking of a five-link underactuated $3 \mathrm{~d}$ bipedal robot," IEEE Transactions on Robotics, vol. 25, no. 1, pp. 37-50, 2009.

[4] S. H. Collins, M. Wisse, and A. Ruina, "A 3-d passive-dynamic walking robot with two legs and knees," International Journal of Robotics Research, vol. 20, pp. 607-615, 2001.

[5] T. Fukuda, M. Doi, Y. Hasegawa, and H. Kajima, Fast Motions in Biomechanics and Robotics, ser. Lecture Notes in Control and Information Sciences. Heidelberg, Germany: Springer, 2006, ch. Multi-Locomotion Control of Biped Locomotion and Brachiation Robot, pp. 121-145.

[6] A. Goswami, "Postural stability of biped robots and the foot rotation indicator (fri) point," International Journal of Robotics Research, vol. 18, no. 6, pp. 523-533, 1999.

[7] J. W. Grizzle, G. Abba, and F. Plestan, "Asymptotically stable walking for biped robots: Analysis via systems with impulse effects," IEEE Transactions on Automatic Control, vol. 46, no. 1, pp. 51-64, 2001.

[8] A. D. Kuo, "Stabilization of lateral motion in passive dynamic walking," International Journal of Robotics Research, vol. 18, no. 9, pp. 917-930, 1999.

[9] J. E. Marsden and T. S. Ratiu, Introduction to Mechanics and Symmetry, ser. Texts in Applied Mathematics. Springer, 1999, vol. 17.

[10] B. Morris and J. W. Grizzle, "A restricted Poincaré map for determining exponentially stable periodic orbits in systems with impulse effects: Application to bipedal robots," in 44th IEEE Conference on Decision and Control and European Control Conference, Seville, Spain, 2005.

[11] R. M. Murray, Z. Li, and S. S. Sastry, A Mathematical Introduction to Robotic Manipulation. Taylor \& Francis/CRC, 1994.

[12] J. E. Pratt and G. A. Pratt, "Exploiting natural dynamics in the control of a $3 \mathrm{~d}$ bipedal walking simulation," in International Conference on Climbing and Walking Robots (CLAWAR99), Portsmouth, United Kingdom.

[13] S. Sastry, Nonlinear Systems: Analysis, Stability and Control. Springer-Verlag, 1999.

[14] R. W. Sinnet and A. D. Ames, "2d bipedal walking with knees and feet: A hybrid control approach," in Joint 48th IEEE Conference on Decision and Control and 28th Chinese Control Conference, Shanghai, P.R. China, 2009.

[15] — , "Functional Routhian reduction applied to a $3 \mathrm{~d}$ bipedal robot with knees and feet," Texas A\&M University, Tech. Rep., 2009.

[16] E. R. Westervelt, J. W. Grizzle, C. Chevallereau, J. Choi, and B. Morris, Feedback Control of Dynamic Bipedal Robot Locomotion. Taylor \& Francis/CRC, 2007.

[17] M. Wisse, A. L. Schwab, and R. Q. vd. Linde, "A 3d passive dynamic biped with yaw and roll compensation," vol. 19, no. 3, pp. 275-284, 2001.

[18] Online content http://www1. mengr.tamu.edu/aames/code. 